\title{
Targeting Senescent Cells: Possible Implications for Delaying Skin Aging: A Mini-Review
}

\author{
Michael C. Velarde ${ }^{a, b}$ Marco Demaria ${ }^{c}$ \\ ${ }^{a}$ Buck Institute for Research on Aging, Novato, Calif., USA; ${ }^{b}$ Institute of Biology, University of the Philippines \\ Diliman, Quezon City, Philippines; ' ${ }^{C}$ European Institute for the Biology of Aging (ERIBA), University Medical Center \\ Groningen (UMCG), Groningen, The Netherlands
}

\section{Key Words}

Cellular senescence - Wound healing .

Senescence-associated secretory phenotype $\cdot$ Skin regeneration · Aging - Immune system - Therapy · Drugs . DNA damage $\cdot$ Mitochondria

\begin{abstract}
Senescent cells are induced by a wide variety of stimuli. They accumulate in several tissues during aging, including the skin. Senescent cells secrete proinflammatory cytokines, chemokines, growth factors, and proteases, a phenomenon called senescence-associated secretory phenotype (SASP), which are thought to contribute to the functional decline of the skin as a consequence of aging. Due to the potential negative effects of the SASP in aged organisms, drugs that selectively target senescent cells represent an intriguing therapeutic strategy to delay aging and age-related diseases. Here, we review studies on the role of senescent cells in the skin, with particular emphasis on the age-related mechanisms and phenotypes associated with excessive accumulation of cellular senescence. We discuss the aberrant behavior of senescent cells in aging and how the different signaling pathways associated with survival and secretion of senescent cells can be engaged for the development of targeted therapies.

(C) 2016 The Author(s)

Published by S. Karger AG, Basel
\end{abstract}

\section{KARGER}

E-Mail karger@karger.com www.karger.com/ger

\section{C) 2016 The Author(s) \\ Published by S. Karger AG, Basel 0304-324X/16/0625-0513\$39.50/0}

This article is licensed under the Creative Commons AttributionNonCommercial-NoDerivatives 4.0 International License (CC BYNC-ND) (http://www.karger.com/Services/OpenAccessLicense). Usage and distribution for commercial purposes as well as any distribution of modified material requires written permission.

\section{Introduction}

Cellular senescence is a tumor-suppressive mechanism wherein cells are permanently growth arrested even in the presence of strong mitogenic signals [1]. Cells are induced to senescence by a wide variety of cellular perturbations, including nuclear DNA damage and mitochondrial dysfunction $[1,2]$. Senescent cells are identified by several nonexclusive markers, such as persistent elevated expression of the cell cycle inhibitors p $21^{\mathrm{WAF} 1}$ and $\mathrm{p} 16^{\mathrm{INK} 4 \mathrm{~A}}$ and of DNA damage response proteins, increased activity of a lysosomal enzyme termed senescence-associated $\beta$-galactosidase, loss of nuclear HMGB1, decreased expression of lamin B1, and different chromatin-remodeling events [1]. Moreover, senescent cells are characterized by the secretion of several proinflammatory factors, a phenomenon called senescence-associated secretory phenotype (SASP) [3]. The accumulation of senescent cells with age is thought to contribute to impaired tissue homeostasis and to different age-related diseases [4]. Lack of cell proliferation in senescent cells hampers the ability of tissues to regenerate after chronic and persistent injury, resulting in tissue damage. The proinflammatory and tissue-remodeling activities of the SASP also create chronic inflammation and alter tissue structure, which are the two main causes of age-related pathology 
[4]. One fascinating hypothesis is that senescent cells might contribute in a cell and non-cell autonomous fashion to skin aging. Skin aging is associated with several pathologies, including lower protection from pathogens, increased irritation, loss of insulation, delayed wound healing and susceptibility to cancer, among others. Here, we summarize the evidence of the presence of senescent cells in the skin, and the potential for pharmaceutical interventions that eliminate the negative effects of senescent cells as methods to delay skin aging.

\section{Senescent Cells in Skin Aging}

Epithelial tissues in humans are constantly renewing, and the skin represents the gold standard example of an epithelial tissue continuously regenerating [5]. Persistent mitogenic signals lead to replicative senescence. Hence, it is not surprising that the number of senescent cells increases with age in the epidermal compartment of the skin [6]. Since proliferation of stem and differentiated cells is a major contributor to skin renewal, the accumulation of an excessive number of senescent cells may cause impairments in tissue regeneration with age [7]. The overexpression of $\mathrm{p} 16^{\mathrm{INK} 4 \mathrm{~A}}$ in young human keratinocytes (isolated from 30 to 40 -year-old patients) results in cellular senescence and in an atrophic layer of epidermis in organotypic cultures, reminiscent of that formed by old keratinocytes (isolated from 53 to 66-year-old patients) [8]. Interestingly, reducing $\mathrm{p} 16^{\mathrm{INK} 4 \mathrm{~A}}$ expression in these old keratinocytes restored the normal thickness of the epidermis, similar to that formed by young keratinocytes. In mice, cellular senescence in the epidermis, caused by persistent mitochondrial damage and dysfunction, is also associated with epidermal thinning with age $[9,10]$.

Senescent cells can also act in a non-cell autonomous fashion through the secretion of several cytokines, chemokines, growth factors, and proteases, a phenomenon described as SASP. In recent years, different laboratories have shown the importance of transiently induced senescent cells and their secretory phenotype to promote optimal cutaneous wound healing. The removal or absence of these short-lived senescent cells during wound healing in young mice impairs granulation, reduces the number of myofibroblasts, results in fibrosis, and delays the time of wound closure $[11,12]$. Thus, the presence of these shortlived senescent cells contributes to proper and timed tissue repair.

Secretion of several proinflammatory and tissue-remodeling factors by senescent cells might contribute to the loss of tissue homeostasis and unbalanced tissue structure [4]. While short-lived senescent cells may act as positive regulators of wound healing, the presence of long-lived senescent cells may exacerbate pathological diseases in the skin. Indeed, the chronic secretion of MMPs by senescent cells might be an important contributor to the degradation of collagen and other extracellular matrix components in the dermal connective tissue, a hallmark of skin aging [13]. Moreover, chronic wounds are associated with the presence of long-lived senescent cells in wound areas $[14,15]$. A persistently elevated number of senescent cells may disrupt cell signaling responses and prevent wound repair to progress through the different stages of the healing process, one of the main features of chronic wounds [16]. Consistent with this idea, cellular senescence also persists in skin fibroblasts after exposure to DNA-damaging agents, such as X-rays, ultraviolet light, and cigarette smoke, resulting in the secretion of factors that may contribute to aging phenotypes $[17,18]$. Increased cellular senescence has also been observed in the skin of patients with different accelerated-aging phenotypes, including Werner syndrome, xeroderma pigmentosum, and Hutchinson-Gilford progeria syndrome [19]. These hereditary disorders are associated with defects in DNA damage repair or nuclear organization that may explain the high frequency of senescent cells in these patients.

\section{Eliminating Senescent Cells}

The impact of senescent cells on animal pathology was directly demonstrated when eliminating senescent cells through a suicide gene in a premature aging mouse model reduced selected age-related pathologies such as sarcopenia, cataracts, and loss of subdermal adipose tissue [20]. Interfering with senescent cells may be beneficial for the overall health of the animal, and the development of specific interventions that target senescent cells may serve as a therapy to delay aging, including skin pathologies. This strategy can be achieved using three different approaches: (1) selective induction of cell death; (2) improvement of the immune system, and (3) inhibition of the SASP (fig. 1).

\section{Selective Induction of Cell Death}

Two different papers reported efficient strategies to specifically eliminate senescent cells. In the first report, Di Mitri and Alimonti [21] used a metabolic approach based on increased glucose uptake and ATP production 
Fig. 1. Eliminating senescent cells and inhibiting the SASP as therapeutic strategy to slow down skin aging. Different drugs have been demonstrated to interfere with signaling pathways that are specific to senescent cells. These drugs show specificity in blocking signals associated with cell survival or secretion of senescent cells, leading to selective cell death or inhibition of the SASP. Another potential approach to target senescent cells relies on enhancing the clearance or increasing the number of NK cells and $\mathrm{T}$ cells.

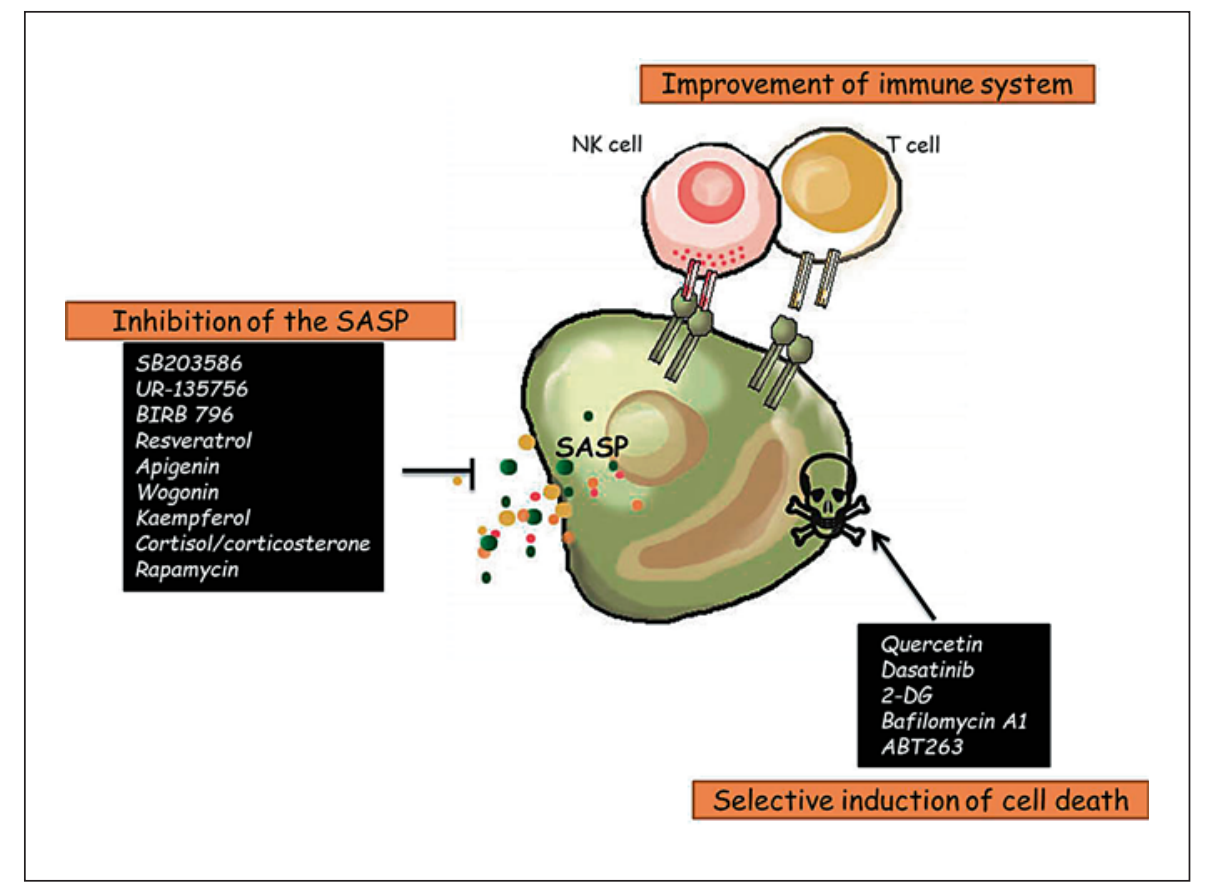

of chemotherapy-induced senescent cells. Senescent cells are induced by standard anti-cancer genotoxic therapies, but despite their ability to improve long-term outcomes, they are also thought to promote several potential harmful properties, particularly through the SASP. Mice bearing lymphomas showed therapy-induced senescence when challenged with the cytotoxic drug cyclophosphamide. Induction of senescence was followed by a hypermetabolic phenotype, which included enhanced glycolysis, fatty acid catabolism, ATP-boosting oxidative phosphorylation, lysosomal protein degradation, and autophagy. Importantly, interference with this aberrant metabolism by treatment with either 2-DG, a false substrate for the glycolytic metabolism, or bafilomycin A1, a specific inhibitor of the lysosomal V-ATPases, was sufficient to reduce the survival of therapy-induced senescent cells and to improve survival of chemotherapy-treated mice bearing lymphomas.

Another recent paper reported the specific elimination of senescent cells upon treatment with the drugs dasatinib and quercetin [22]. Dasatinib, which is known to inhibit ephrin B (EFNB)-dependent suppression of apoptosis, preferentially decreases viability and increases cell death in senescent compared to nonsenescent human preadipocytes. Quercetin, which inhibits phosphoinositide 3kinase, preferentially induced cell death in senescent relative to nonsenescent HUVEC. Combination of dasatinib and quercetin reduced senescent cells in fat and liver tissues of old mice, as well as in muscle and fat tissues of irradiated mice. Moreover, this combined treatment alleviated several age-related pathologies, such as impaired cardiovascular function and extended health span of the $\mathrm{Erccl}^{-/ \Delta}$ progeroid mouse model, supporting the therapeutic potential of eliminating senescent cells at old age.

The ability of dasatinib and quercetin to selectively eliminate senescent cells suggests that there are different signaling pathways which regulate cell survival in senescent versus nonsenescent cells. Dasatinib and quercetin inhibit EFNB and phosphoinositide 3-kinase signaling pathways, respectively. Consistent with this, knockdown of mRNA transcripts to EFNB1 and EFNB3 ligands and phosphatidylinositol-4,5-bisphosphate 3-kinase delta catalytic subunit (PIK3CD) triggered cell death in senescent cells with little effect on proliferating, quiescent, and differentiated nonsenescent cells [22], demonstrating the importance of these pathways in targeting the elimination of senescent cells. Knockdown of other mRNA transcripts, such as $21^{\mathrm{WAF} 1}$, plasminogen-activated inhibitor-2 (PAI-2), and BCL-xL, also selectively killed senescent relative to nonsenescent cells [22]. Identifying molecules that can target these pathways may help classify senolytic specific agents with minimal effect on nonsenescent cells. Indeed, ABT263, which targets both the antiapoptotic proteins BCL-xL and BCL-2, has been 
recently shown to specifically induce apoptosis in senescent cells [23].

\section{Improvement of the Immune System}

Another possible way of eliminating senescent cells is to increase the number and/or activity of immune cells that can selectively recognize and remove senescent cells. Indeed, natural killer (NK) cells and T cells trigger cytolytic responses on senescent cells [24]. Moreover, the CD4+ T cell-mediated adaptive immune response initiates an immune-dependent clearance of senescent cells termed senescence surveillance [25]. The decline in immune function with age is consistent with the high number of senescent cells at old age [4], further supporting the idea that the immune system may limit the number of senescent cells through clearance of these cells. Hence, it may be worth developing a strategy that boosts the immune cells capable of specifically eliminating senescent cells.

\section{Inhibition of the SASP}

Apart from the removal of senescent cells, decreasing the effect of the SASP may potentially be an alternative strategy to dampen the negative effects of long-lived senescent cells. Overexpression of oncogenic RAS, which causes genotoxic stress and cellular senescence, is a strong inducer of the SASP [3]. RAS activation can trigger a phosphorylation cascade involving activation of the mitogen-activated protein kinase (MAPK) family of proteins. Consistent with this, the MAPK family member p38MAPK is an important regulator of the SASP [26]. Treatment with the p38MAPK inhibitor SB203580 potently suppressed SASP expression in senescent cells. Two next-generation p38 inhibitors UR-13756 and BIRB 796 , which are more selective and specific compared to SB203580, can also dampen the SASP [27]. BIRB 796 has reached phase III clinical trials, suggesting that $\mathrm{p} 38 \mathrm{MAPK}$ may be a potential therapeutic target to suppress the SASP in vivo. Inhibition of MK2 kinase (also MAPKAPK2), a downstream target of p38MAPK, can also inhibit the SASP [27]. MK2 is implicated in phosphorylation of the RNA-binding protein ZFP36L1, which degrades transcripts of several SASP components. Hence, MK2 kinase inhibition is also being investigated as a potential pharmacological strategy to dampen senescenceassociated inflammation.

p38MAPK activation of the SASP is largely due to an increase in nuclear factor- $\kappa \mathrm{B}(\mathrm{NF}-\kappa \mathrm{B})$ transcriptional activity independently of the canonical DNA damage response [26]. NF- $\mathrm{KB}$ is thought to be an important induc- er of the SASP. It is one of the transcription factors that re-enforces the SASP response [28]. Many signaling pathways that modulate the SASP seem to converge with NF$\kappa B$. For example, accumulation of GATA4 in senescent cells, as a consequence of its impaired degradation by p62-mediated autophagy, leads to activation of NF- $\kappa B$ and subsequent upregulation of the SASP [29]. Unlike p38MAPK, which is independent of the DNA damage response, GATA4-induced SASP is dependent on the DNA damage response, but independent of p53 and p16 ${ }^{\mathrm{INK} 4 \mathrm{~A}}$ [29]. Chronic resveratrol treatment also dampens the SASP [30], likely through its ability to decrease I $\kappa$ B kinase activity, which leads to decreased I $\kappa \mathrm{B} \alpha$ phosphorylation and subsequent NF- $\kappa \mathrm{B}$ activation [31]. Other natural compounds, such as apigenin, wogonin, and kaempferol, inhibit the SASP by blocking I $\mathrm{\kappa} \mathrm{C}$ expression and reducing NF- $\kappa B$ activity [32].

Another important activator of NF- $\kappa \mathrm{B}$ and the SASP is interleukin-1 alpha (IL1A or IL-1 $\alpha$ ). Increased expression of plasma membrane-bound IL1A in senescent cells activates the plasma membrane-bound IL-1 receptor in juxtacrine cells, resulting in upregulation of several transcripts associated with inflammation [33]. IL1A-blocking antibody or knockdown of IL1A by RNA interference diminished SASP expression in senescent cells [33]. Moreover, exogenous IL1A restored IL-6 and IL-8 secretion to IL1A-knockout cells, further supporting a direct role of IL1A in SASP regulation [33]. Compounds that disrupt IL1A receptor signaling may serve as a strategy to dampen the SASP. Indeed, the glucocorticoids cortisol and corticosterone suppress IL1A signaling in senescent cells and decrease expression of the SASP components, but cotreatment with recombinant IL1A reestablishes the SASP [34]. Overexpressing the microRNA miR-146a/b, which knocks down the levels of the IL1A downstream target IRAK1 (also an upstream regulator of NFkB), resulted in decreased SASP expression [35]. Treatment with the mTOR inhibitor rapamycin also caused downregulation of IL-6 and other SASP factors, partly due to suppressing translation of the membrane-bound IL1A [36]. Additionally, rapamycin-induced SASP is also thought to result from inhibition of MK2 translation and inactivation of ZFP36L1 activity [37].

Other regulators of the SASP include p53, C/EBP $\beta$, Sirt1, and NAD+/NADH redox balance. Functional loss of the 553 protein exacerbates the SASP $[3,38]$. C/EBP $\beta$ is implicated in upregulating the SASP and may cooperate with IL-6 to amplify the SASP $[28,39]$. Sirt1 suppresses SASP expression through histone deacetylation at the promoter regions of SASP factors [40]. Interestingly, 
Sirt1 is also pharmacologically activated by resveratrol [41], which is capable of dampening the SASP [30]. The activation of Sirt1 by resveratrol should be explored further, particularly with regard to p53 inhibition [42]. Imbalance in NAD+/NADH redox due to impaired mitochondrial activity results in reduced expression of selected SASP factors [43]. Designing drugs that target SASP regulators may help in developing pharmaceutical therapies to limit the negative side effects of the SASP.

\section{Conclusion}

Removal of senescent cells and reducing the SASP are being considered as therapeutic strategies to delay the onset of age-related pathologies. Several drugs have already been identified that selectively target senescent cells (fig. 1). Some of them might have toxic effects when administered systemically for a long period of time: for example, ABT263 can cause thrombocytopenia in patients treated with an oral form of the compound. However, the toxicity might be highly reduced by developing drugs for topical treatment, an approach that would be suitable for skin interventions. The contribution of senescent cells to skin function is complex because they may be both beneficial and detrimental depending on the context; it is still unclear whether senolytic drugs will delay skin aging [6]. Senescent cells are important for proper wound healing through their secretion of the SASP factor PDGF-AA and through their capacity to limit fibrosis [12], while chronic induction of cellular senescence through mitochondrial dysfunction may contribute to stem cell loss with age [10]. Hence, proper testing of dosage and timing must be investigated to determine if these drugs would indeed reduce the negative impact on skin aging. Nonetheless, the possibility of selectively targeting senescent cells through pharmacological interventions posits a potential new solution to the functional decline associated with skin aging.

\section{Disclosure Statement}

The authors have no conflicts of interest to disclose.

\section{References}

1 Sharpless NE, Sherr CJ: Forging a signature of in vivo senescence. Nat Rev Cancer 2015;15: 397-408.

2 Ziegler DV, Wiley CD, Velarde MC: Mitochondrial effectors of cellular senescence: beyond the free radical theory of aging. Aging Cell 2015;14:1-7.

3 Coppe JP, Patil CK, Rodier F, Sun Y, Munoz DP, Goldstein J, Nelson PS, Desprez PY, Campisi J: Senescence-associated secretory phenotypes reveal cell-nonautonomous functions of oncogenic RAS and the p53 tumor suppressor. PLoS Biol 2008;6:2853-2868.

4 van Deursen JM: The role of senescent cells in ageing. Nature 2014;509:439-446.

5 Flores ER, Halder G: Stem cell proliferation in the skin: alpha-catenin takes over the hippo pathway. Sci Signal 2011;4:pe34.

-6 Demaria M, Desprez PY, Campisi J, Velarde MC: Cell autonomous and non-autonomous effects of senescent cells in the skin. J Invest Dermatol 2015;135:1722-1726.

7 Signer RA, Morrison SJ: Mechanisms that regulate stem cell aging and life span. Cell Stem Cell 2013;12:152-165.

$\checkmark 8$ Adamus J, Aho S, Meldrum H, Bosko C, Lee JM: p16INK4A influences the aging phenotype in the living skin equivalent. J Invest Dermatol 2014;134:1131-1133.
9 Velarde MC, Flynn JM, Day NU, Melov S, Campisi J: Mitochondrial oxidative stress caused by Sod 2 deficiency promotes cellular senescence and aging phenotypes in the skin. Aging 2012;4:3-12.

10 Velarde MC, Demaria M, Melov S, Campisi J: Pleiotropic age-dependent effects of mitochondrial dysfunction on epidermal stem cells. Proc Natl Acad Sci USA 2015;112: 10407-10412.

11 Jun JI, Lau LF: The matricellular protein CCN1 induces fibroblast senescence and restricts fibrosis in cutaneous wound healing. Nat Cell Biol 2010;12:676-685.

12 Demaria M, Ohtani N, Youssef SA, Rodier F, Toussaint W, Mitchell JR, Laberge RM, Vijg J, Van Steeg H, Dolle ME, Hoeijmakers JH, de Bruin A, Hara E, Campisi J: An essential role for senescent cells in optimal wound healing through secretion of PDGF-AA. Dev Cell 2014;31:722-733.

13 Fisher GJ, Kang S, Varani J, Bata-Csorgo Z, Wan Y, Datta S, Voorhees JJ: Mechanisms of photoaging and chronological skin aging. Arch Dermatol 2002;138:1462-1470.

14 Mendez MV, Stanley A, Park HY, Shon K, 20 Phillips T, Menzoian JO: Fibroblasts cultured from venous ulcers display cellular characteristics of senescence. J Vasc Surg 1998;28:876883.
15 Vande Berg JS, Rose MA, Haywood-Reid PL, Rudolph R, Payne WG, Robson MC: Cultured pressure ulcer fibroblasts show replicative senescence with elevated production of plasmin, plasminogen activator inhibitor-1, and transforming growth factor-beta1. Wound Repair Regen 2005;13:76-83.

16 Sen CK, Gordillo GM, Roy S, Kirsner R, Lambert L, Hunt TK, Gottrup F, Gurtner GC, Longaker MT: Human skin wounds: a major and snowballing threat to public health and the economy. Wound Repair Regen 2009;17: 763-771.

17 Shin J, Kim JH, Kim EK: Repeated exposure of human fibroblasts to UVR induces secretion of stem cell factor and senescence. J Eur Acad Dermatol Venereol 2012;26:1577-1580.

18 Yang GY, Zhang CL, Liu XC, Qian G, Deng DQ: Effects of cigarette smoke extracts on the growth and senescence of skin fibroblasts in vitro. Int J Biol Sci 2013;9:613-623.

19 Moriwaki S: Human DNA repair disorders in dermatology: a historical perspective, current concepts and new insight. J Dermatol Sci 2016;81:77-84

20 Baker DJ, Wijshake T, Tchkonia T, LeBrasseur NK, Childs BG, van de Sluis B, Kirkland JL, van Deursen JM: Clearance of p16Ink4apositive senescent cells delays ageing-associated disorders. Nature 2011;479:232-236. 
-21 Di Mitri D, Alimonti A: Non-cell-autonomous regulation of cellular senescence in cancer. Trends Cell Biol 2016;26:215-226.

-22 Zhu Y, Tchkonia T, Pirtskhalava T, Gower AC, Ding H, et al: The Achilles' heel of senescent cells: from transcriptome to senolytic drugs. Aging Cell 2015;14:644-658.

23 Chang J, Wang Y, Shao L, Laberge RM, Demaria M, Campisi J, Janakiraman K, Sharpless NE, Ding S, Feng W, Luo Y, Wang X, AykinBurns N, Krager K, Ponnappan U, HauerJensen M, Meng A, Zhou D: Clearance of senescent cells by ABT263 rejuvenates aged hematopoietic stem cells in mice. Nat Med 2016; 22:78-83.

-24 Krizhanovsky V, Yon M, Dickins RA, Hearn S, Simon J, Miething C, Yee H, Zender L, Lowe SW: Senescence of activated stellate cells limits liver fibrosis. Cell 2008; $134: 657-$ 667.

25 Kang TW, Yevsa T, Woller N, Hoenicke L, Wuestefeld T, et al: Senescence surveillance of pre-malignant hepatocytes limits liver cancer development. Nature 2011;479:547-551.

26 Freund A, Patil CK, Campisi J: p38MAPK is a novel DNA damage response-independent regulator of the senescence-associated secretory phenotype. EMBO J 2011;30:1536-1548.

27 Alimbetov D, Davis T, Brook AJ, Cox LS, Faragher RG, Nurgozhin T, Zhumadilov Z, Kipling D: Suppression of the senescence-associated secretory phenotype (SASP) in human fibroblasts using small molecule inhibitors of p38 MAP kinase and MK2. Biogerontology 2015, Epub ahead of print.

28 Acosta JC, O’Loghlen A, Banito A, Guijarro MV, Augert A, Raguz S, Fumagalli M, Da Costa M, Brown C, Popov N, Takatsu Y, Melamed J, d'Adda di Fagagna F, Bernard D, Hernando E, Gil J: Chemokine signaling via the CXCR2 receptor reinforces senescence. Cell 2008;133:1006-1018.
29 Kang C, Xu Q, Martin TD, Li MZ, Demaria M, Aron L, Lu T, Yankner BA, Campisi J, Elledge SJ: The DNA damage response induces inflammation and senescence by inhibiting autophagy of GATA4. Science 2015;349: aaa5612.

30 Pitozzi V, Mocali A, Laurenzana A, Giannoni E, Cifola I, Battaglia C, Chiarugi P, Dolara P, Giovannelli L: Chronic resveratrol treatment ameliorates cell adhesion and mitigates the inflammatory phenotype in senescent human fibroblasts. J Gerontol A Biol Sci Med Sci 2013;68:371-381.

31 Holmes-McNary M, Baldwin AS Jr: Chemopreventive properties of trans-resveratrol are associated with inhibition of activation of the IkappaB kinase. Cancer Res 2000;60:34773483.

32 Lim H, Park H, Kim HP: Effects of flavonoids on senescence-associated secretory phenotype formation from bleomycin-induced senescence in BJ fibroblasts. Biochem Pharmacol 2015;96:337-348.

33 Orjalo AV, Bhaumik D, Gengler BK, Scott GK, Campisi J: Cell surface-bound IL-1alpha is an upstream regulator of the senescenceassociated IL-6/IL-8 cytokine network. Proc Natl Acad Sci USA 2009;106:17031-17036.

34 Laberge RM, Zhou L, Sarantos MR, Rodier F, Freund A, de Keizer PL, Liu S, Demaria M, Cong YS, Kapahi P, Desprez PY, Hughes RE, Campisi J: Glucocorticoids suppress selected components of the senescence-associated secretory phenotype. Aging Cell 2012;11:569578.

35 Bhaumik D, Scott GK, Schokrpur S, Patil CK, Orjalo AV, Rodier F, Lithgow GJ, Campisi J: MicroRNAs miR-146a/b negatively modulate the senescence-associated inflammatory mediators IL-6 and IL-8. Aging 2009;1:402-411.
36 Laberge RM, Sun Y, Orjalo AV, Patil CK, Freund A, et al: MTOR regulates the pro-tumorigenic senescence-associated secretory phenotype by promoting IL1A translation. Nat Cell Biol 2015;17:1049-1061.

37 Herranz N, Gallage S, Mellone M, Wuestefeld T, Klotz S, Hanley CJ, et al: mTOR regulates MAPKAPK2 translation to control the senescence-associated secretory phenotype. Nat Cell Biol 2015;17:1205-1217.

38 Christy B, Demaria M, Campisi J, Huang J, Jones D, Dodds SG, Williams C, Hubbard G, Livi CB, Gao X, Weintraub S, Curiel T, Sharp ZD, Hasty P: p53 and rapamycin are additive. Oncotarget 2015;6:15802-15813.

39 Kuilman T, Michaloglou C, Vredeveld LC, Douma S, van Doorn R, Desmet CJ, Aarden LA, Mooi WJ, Peeper DS: Oncogene-induced senescence relayed by an interleukin-dependent inflammatory network. Cell 2008;133: 1019-1031.

40 Hayakawa T, Iwai M, Aoki S, Takimoto K, Maruyama M, Maruyama W, Motoyama N: SIRT1 suppresses the senescence-associated secretory phenotype through epigenetic gene regulation. PLoS One 2015;10:e0116480.

41 Howitz KT, Bitterman KJ, Cohen HY, Lamming DW, Lavu S, Wood JG, Zipkin RE, Chung P, Kisielewski A, Zhang LL, Scherer B, Sinclair DA: Small molecule activators of sirtuins extend Saccharomyces cerevisiae lifespan. Nature 2003;425:191-196.

42 Yi J, Luo J: SIRT1 and p53, effect on cancer, senescence and beyond. Biochim Biophys Acta 2010;1804:1684-1689.

43 Wiley CD, Velarde MC, Lecot P, Liu S, Sarnoski EA, Freund AM, Shirakawa K, Lim HW, Davis SS, Ramanathan A, Gerencser AA, Verdin E, Campisi J: Mitochondrial dysfunction induces senescence with a distinct secretory pheonotype. Cell Metab 2016;23:303314 